\title{
Small molecule efflux pump inhibitors in Mycobacterium tuberculosis: a rational drug design perspective
}

\author{
Erika Kapp, Sarel F. Malan, Jacques Joubert and Samantha L. Sampson
}

\begin{abstract}
:
Drug resistance in Mycobacterium tuberculosis (M. tuberculosis) complicates management of tuberculosis. Efflux pumps contribute to low level resistance and acquisition of additional high level resistance mutations through sub-therapeutic concentrations of intracellular antimycobacterials. Various efflux pump inhibitors (EPIs) have been described for $M$. tuberculosis but little is known regarding the mechanism of efflux inhibition. As knowledge relating to the mechanism of action and drug target is central to the rational drug design of safe and sufficiently selective EPIs, this review aims to examine recent developments in the study of EPIs in M. tuberculosis from a rational drug development perspective and to provide an overview to facilitate systematic development of therapeutically effective EPIs. Review of literature points to a reduction in cellular energy or direct binding to the efflux pump as likely mechanisms for most EPIs described for M. tuberculosis. This review demonstrates that, where a direct interaction with efflux pumps is expected, both molecular structure and general physicochemical properties should be considered to accurately predict efflux pump substrates and inhibitors. Non-competitive EPIs do not necessarily demonstrate the same requirements as competitive inhibitors and it is therefore essential to differentiate between competitive and non-competitive inhibition to accurately determine structure activity relationships for efflux pump inhibition. It is also evident that there are various similarities between inhibitors of prokaryotic and eukaryotic efflux pumps but, depending on the specific chemical scaffolds under investigation, it may be possible to design EPIs that are less prone to inhibition of human P-glycoprotein, thereby reducing side effects and drug-drug interactions.
\end{abstract}

\section{Introduction}

Microbial drug resistance is fast becoming a significant risk to global public health. With the emergence of the human immunodeficiency virus (HIV) epidemic, tuberculosis (TB) was re-established as one of the most dangerous communicable diseases of our time. Treatment of TB is hampered by the development of multidrugresistant (MDR) and extensively drug-resistant (XDR) TB. Drug resistance in $M$. tuberculosis can be intrinsic or acquired. Intrinsic resistance is attributed to the unusual structure of the mycobacterial cell wall as well as the natural abundance of 
efflux pumps (EPs) limiting the intracellular accumulation of various classes of antibiotics [1-3]. Acquired resistance can usually be attributed to mutations in chromosomal genes. It has been established that mutations responsible for the resistance to various drugs are often not linked and that multi-drug resistance is not due to a single genetic mutation but rather a result of a combination of multiple different mutations [4]. It is however possible that a complex association exists between mutations associated with one drug and the development of further resistance mutations [1]. Chromosomal alterations or deletions can affect the drug target, bacterial enzymes that activate/ inactivate the drug and influx or efflux of drugs into/from the intracellular environment $[2,4,5]$.

EPs contribute towards intrinsic and acquired mycobacterial resistance through their natural abundance, inducible expression and development of mutations that enhance efflux activity $[2,3]$. Active efflux reduces the intracellular concentration of substrate compounds to sub-therapeutic levels thereby directly conferring resistance to the given substrate and chemical properties. A number of studies successfully suggested SAR or employed computer based drug design of EPIs in bacteria where crystal structures for EPs are available [14]. The limited availability of crystal structures for EPs (particularly in $M$. tuberculosis), large structural diversity of putative EPIs, indiscriminate inhibition of a range of EPs by similar EPIs and limited knowledge relating to the specific interactions with EPs or even EPI mechanism of action, complicates rational drug design of EPIs $[12,13]$. Thus far, authors have employed ligand-based drug design approaches or homology modelling of efflux pumps to suggest preliminary SARs for EPI activity in M. tuberculosis [23, 26, 27].

Efflux-mediated resistance and the development of EPIs is an ongoing research interest of various research groups and original research and review articles are regularly published in this field. In prokaryotic cells, and mycobacteria in particular, there however seems to be a gap in investigations and reviews into the mechanism of action of efflux pump inhibition [3, 17, 28, 29]. One could argue that whole cell phenotypic screenings of compound libraries have been, and remain, effective identifiers of antimycobacterials [23] and EPIs, and that information relating to specific mechanisms of action or inhibition of efflux is not essential for this method of drug discovery. Rational drug development, as an alternative (or contributor to the aforementioned approach), could produce therapeutically active compounds that demonstrate suitable host pharmacokinetic properties, acceptable safety profiles and possibly exhibit reduced tendencies for development of resistance. As knowledge relating to the mechanism of action and drug target is central to the rational drug design approach, this review aims to examine recent developments in the study of EPIs in $M$. tuberculosis from a rational drug development perspective and provide an overview/platform to facilitate systematic development of safe and therapeutically effective EPIs. 


\section{Efflux pump inhibitors in Mycobacterium tuberculosis}

Various confirmed and putative EPI have been evaluated in M. tuberculosis over the past 2 decades. A paper by Kourtesi and colleagues describe a number of assays employed in the screening of EPI activity in bacteria [12]. A popular phenotypic assay for possible efflux inhibition in mycobacteria is administration of the putative EPI with known antimycobacterials and assessment of the reduction in MIC or fold potentiation of the antimycobacterial. Some studies build on this MIC assessment by characterizing EPs that are overexpressed in the specific strains under evaluation and more recently performing fractional inhibitory concentration indices (FICI) assays that predict possible synergism between the putative EPI and the substrate in question. Researchers also utilize ethidium bromide (EB) and other fluorescent efflux pump substrates or dyes in functional assays of EP activity in mycobacteria. It is however pertinent to note that (1) reduction of the MIC or synergism observed with antimycobacterials may be obtained by mechanisms other that EP inhibition; (2) characterization of overexpressed EPIs does not confirm that the compound under evaluation interacts with all/any of the pumps identified; (3) EB assays measure accumulation of EB which reflects the balance between cell wall permeability and efflux activity and it may therefore be difficult to distinguish by which mechanism the compound under evaluation influences the accumulation of EB. However, when viewed together, these assays give a good indication of the likelihood of EP inhibitory activity and may indicate possible EP targets of the compound under evaluation. It is also important to note that extensive variation exists in the expression of EPs in different strains of $M$. tuberculosis and that experimental conditions, the substrate being evaluated and the concentration [30] of the EPI being used, to a large extent influences the activation of EPs and inhibition of efflux. That being said, Table 1 provides a general classification of the more prominent putative EPIs that have been used in- or evaluated for activity in EPI assays in M.tuberculosis.

\section{Mechanisms by which efflux is mediated}

Various mechanisms have been suggested for EP inhibition in prokaryotic cells. Interference with energy required for active efflux of substrates feature prominently but a direct interaction with efflux pumps, likely competitive inhibition, has also been suggested. 
Table 1. Putative efflux pump inhibitors for Mycobacterium tuberculosis.

\begin{tabular}{|c|c|c|}
\hline Efflux Pump Inhibitor Class & Classification & References \\
\hline \hline Verapamil \& analogues & Ion channel inhibitor & {$[18,27-30,33,34,37-42]$} \\
\hline Phenothiazine derivatives & Antipsychotic; Ion channel inhibitor & {$[23,29,30,35,45-49,51,54,55,59]$} \\
\hline PaßN & RND-type efflux pump inhibitor & {$[25,89-92]$} \\
\hline Timcodar & P-gp inhibitor & {$[78-84]$} \\
\hline Reserpine & Antihypertensive and antipsychotic, inhibitor of transport pumps and ion \\
channels & {$[26,27,30,63-70]$} \\
\hline Piperine & Activates TRPV ion channels; enzyme inhibitor, P-gp inhibitor. & {$[12,71,72-76,81]$} \\
\hline Berberine & Efflux pump substrate; potentiates effects of antibiotics and antifungals & {$[77,84]$} \\
\hline Tetrandrine & L-type calcium channel antagonist; P-gp inhibitor & \\
\hline
\end{tabular}

Energy for many cell processes including the activity of active transport systems is dependent on the ability of the cell to maintain transmembrane potential and generate and use ATP [31, 32]. Any compound that targets the electron transport chain and interferes with energy production of a cell directly affects cell viability but may also interfere with the ability of the cell to actively extrude compounds from the intracellular environment, thereby increasing the intracellular concentration of coadministered compounds $[8,22]$. Proton motive force is the direct energy source for all secondary active transport pumps but also plays an important part in the production of ATP as utilized by the ABC super family to drive active efflux. The ability of a cell to maintain membrane potential is vital to generating sufficient PMF and energy for cellular processes. As transmembrane electrochemical potential is a function of the electrostatic or charge difference (electrical gradient) as well as the concentration difference of specific ions (chemical gradient) across the cell membrane, the action of ionophores, but also ion channel blockers, influence cellular energy levels [22, 31, 33]. Compounds that inhibit metabolic enzymes that yield hydronium ions required for the maintenance of PMF may also affect efflux in a similar fashion [20].

Various current and prospective antimycobacterials target the generation of proton motive force and ATP [8] and a number of putative EPIs have also been shown to deplete cellular energy $[8,34,35]$. As there are many similarities in the mechanisms of generation of cellular energy across cell types, selective toxicity to the cells that are targeted is a significant concern in the use and future development of therapeutic agents that utilize this mechanism of action. Even though protonophores are used as EPIs in some in vitro assays, their indiscriminate mechanism of action and/or host toxicity at doses required to inhibit efflux complicates (but does not exclude) development of these and similar compounds into viable therapeutic options [36]. Ion channel blockers instead, interact with specific channels to alter physiological movement of ions across membranes. Although blockage of ion channels may influence membrane potential and generation of cellular energy [22] as do protonophores, the mechanism by which this is achieved is more specific and eases 
systematic development of compounds with selective toxicity in cellular energy depletion. Depending on their potency and the role of the pumps being influenced, ion channel inhibitors could have direct antimicrobial activity and/or act synergistically with anti-mycobacterials as part of combination therapy [35].

Alternatively, a direct interaction with efflux pumps has also been suggested as a mechanism of action for efflux pump inhibition. This type of inhibition could be competitive or non-competitive in nature. Non-competitive inhibition could block access to substrate binding cavity or interfere with efflux pump conformation through interaction with an alternative binding site. In the case of competitive inhibition, the inhibitor would likely occupy the same binding cavity as the substrate. It could therefore be postulated that molecular and physicochemical properties for these competitive inhibitors may overlap with substrates for the specific efflux pump.

The molecular mechanism of action for substrate recognition and subsequent efflux depends on the efflux pump type, likely with additional variation between various pumps in that category, and within different species of bacteria. Fundamentally, the substrate would migrate or gain access to the efflux pump binding pocket and interact with various amino acids within this binding site, thereby initiating a change in conformation of the protein domains(s) with resulting extrusion of the substrate from the cytoplasm. The rate and extent of efflux would amongst others depend on the availability of energy (PMF or ATP) to drive active efflux. In certain efflux pump types, protonation or deprotonation of a number of ionizable amino acid residues within the various pump domains likely play a role in the extrusion process [9]. The suggested good recognition of amphiphilic compounds as substrates of efflux pumps may point to the need for both lipophilic interactions between the substrate and the binding cavity residues with hydrophobic side chains as well as interactions with residues with polar or charged side chains. The latter may influence the extent of ionization of various amino acids and thereby directly link to changes in the efflux pump conformation. Depending on the importance of ionic or polar interactions in efflux function as well as substrate and inhibitor recognition, the $\mathrm{pKa}$ of the compound and $\mathrm{pH}$ of the immediate environment may affect efflux to a greater or lesser extent. The non-specific nature of substrate recognition by a number of efflux pumps may result from the involvement of different residues within the binding pocket in the binding of diverse substrates [9, 14, 25]. Although there may be overlap between species and efflux pump types, the exact nature of substrate-efflux pump interactions and proceeding conformational changes are specific to an efflux pump and the particular substrate (or inhibitor) involved. These interactions are often investigated in silico where crystal structures of the efflux pump proteins are available [14]. It should also be possible to successfully predict interactions with the use of carefully constructed homology models. The lack of crystal structures and homology models for efflux pumps in Mtb thus largely contribute to limited knowledge pertaining to the molecular mechanism of action of EPIs in Mtb. 
Competitive inhibition has been demonstrated for various P-glycoprotein inhibitors in mammalian cells as well as for inhibitors of a number of efflux family types in bacterial cells. In $M$. tuberculosis a direct interaction with efflux pumps has been suggested as a mechanism of efflux inhibition for verapamil [27] and piperine [26]. Both publications suggest that the altered efflux activity of mycobacterial cells observed in the respective assays is due to an interaction of the evaluated derivatives with the MFS-type efflux pump Rv1258c. In M. tuberculosis Rv1258c has been extensively implicated in drug resistance to various antimycobacterials [27, 37].

\section{Verapamil analogues}

Verapamil is an inhibitor of L-type voltage dependent calcium channels and is used clinically to manage hypertension, cardiac arrhythmias, angina pectoris and cluster headaches [33, 38]. It is a phenylalkylamine derivative with one chiral carbon yielding the two stereoisomers, $R$ - and $S$ - verapamil (Fig. 1). Clinically, verapamil is administered as a racemate even though different therapeutic properties and potencies have been described for the respective enantiomers [39, 40].

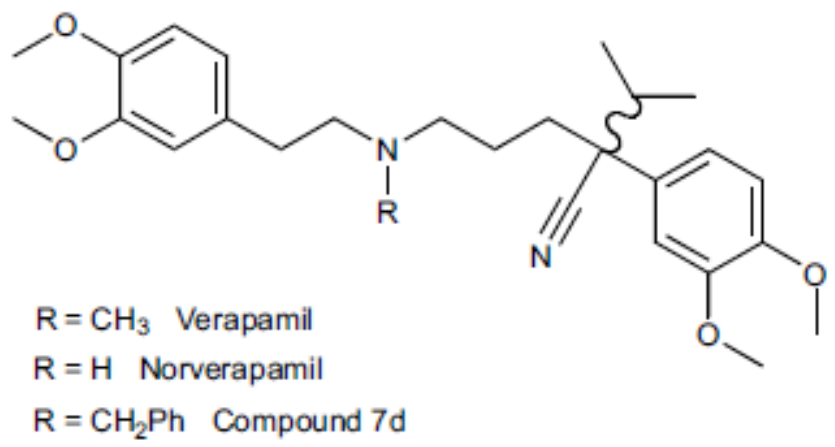

Fig. (1). Chemical structures of verapamil and derivatives.

The ability of verapamil to block efflux pumps across cell types has been well described in literature. Numerous studies have demonstrated that verapamil blocks efflux pumps in $M$. tuberculosis and is able to decrease resistance to- and reduce the minimum inhibitory concentration of various antimycobacterials [27, 29, 30 41, 42]. Verapamil inhibits efflux of several structurally unrelated compounds, seemingly extruded by both ATP-dependent and a range of proton motive force driven efflux pumps.

The mechanism of prokaryotic efflux pump inhibition of verapamil has not been fully elucidated. Some studies suggest a direct interaction with efflux pumps [27] while others propose a depletion of energy required for active efflux by ion channel inhibitors in general $[18,28,29,34]$. Verapamil is an amphipathic molecule with a tertiary amine which is protonated at physiological $\mathrm{pH}$ (pKa 9.68 Chemaxon). As is the case with local anaesthetics with similar physicochemical properties, accumulation of verapamil within the lipid membrane may increase membrane permeability and 
interfere with the function of membrane proteins [43]. It has been suggested that the natural substrate pool for SMR and MFS efflux pumps comprise largely out of amphipathic cations [21] which may point to a certain extent of competitive antagonism of efflux. It is thus possible that the mechanism of efflux inhibition may be a combination of the methods suggested above and that the experimental conditions, cell type, the substrate as well as specific EPs expressed and activated may to a large extent determine the mechanism through which efflux is inhibited.

\subsection{Cellular Energy}

An article recently published by Machado and colleagues confirmed EPI activity of a range of therapeutic ion channel blockers (verapamil, chlorpromazine, thioridazine, flupenthixol and haloperidol) in an EB efflux assay in M. tuberculosis [35]. Of the compounds evaluated, verapamil showed the most potent inhibition of efflux. The same study demonstrated that these compounds resulted in a rapid reduction in intracellular ATP levels in $M$ tuberculosis. This depletion in energy could result from interference with membrane potential and generation of ATP. It might be worth noting that, in addition to interference with membrane potential, compounds that interfere with calcium movement and transport (e.g. verapamil and phenothiazines) may interfere with calcium dependent generation of energy from hydrolysis of ATP and thereby activity of efflux pumps $[28,34]$.

\subsection{Direct Interaction with Efflux Pumps}

The use of verapamil as a chemosensitizer in multi-drug resistant cancer cell lines has been extensively studied. The suggested mechanism of action in eukaryotic cells is via competitive antagonism of efflux through irreversible binding to the $\mathrm{p}$ glycoprotein transporter. Studies suggest that efflux pump inhibition is likely independent of calcium channel blocking activity [44]. In M. tuberculosis, Adams and colleagues demonstrated that, despite their reduced cardiac activity, $R$-verapamil and norverapamil (pKa 10.29 Chemaxon) were as effective as verapamil in reducing macrophage-induced drug tolerance in an infected macrophage model. This would suggest that the mechanism of reversal of drug tolerance is not directly linked to the mammalian calcium channel activity of verapamil analogues [30].

Although many studies were able to characterize specific EPs that were overexpressed in $M$. tuberculosis strains where verapamil was able to reduce the MIC of- or reverse resistance to antimycobacterial agents (see Table 1), few studies have demonstrated a direct interaction with any of the overexpressed EPs. One of these studies by Singh and colleagues evaluated the effect of verapamil and analogues on the MIC of rifampicin in $M$. tuberculosis. The authors suggested preliminary structure activity relationships for the interaction of verapamil with the efflux protein Rv1258c [27] that encodes for a secondary-active transporter from the major facilitator superfamily. Singh and colleagues synthesized a series of verapamil analogues and calculated fractional inhibitory concentration indices (FICI) to evaluate possible synergistic activity of these compounds with rifampicin against the laboratory strain H37Rv. Docking studies, using 
a validated homology model of Rv1258c, were performed with the derivatives demonstrating the best synergistic activity $(7 \mathrm{~d}$, Fig. 1, FICI $=0.3$ versus verapamil FICI 0.5) and the binding affinity and residue interactions of $7 \mathrm{~d}$ was compared to that of piperine, reserpine and verapamil. The authors were able to demonstrate favourable interactions with Rv1258c binding sites and multiple interactions with a combination of residues that also featured in the binding of verapamil, reserpine and piperine respectively. Compound $7 \mathrm{~d}$ was shown to interact amongst others with the same arginine and glycine functions as piperine whereas verapamil formed hydrogen bonds with the same arginine amino acid as well as an additional tryptophan function [27]. Sharma and colleagues used a similar method to prepare an Rv1258c model for docking of piperine analogues as discussed in more detail later in the review [26].

Although not yet used clinically due to uncertainties relating to systemic side effects and drug-drug interactions with commonly used antimycobacterials, various authors have identified verapamil and verapamil analogues as good lead compounds for development of therapeutically useable EPIs.

\section{Phenothiazine analogues}

The phenothiazine class of compounds exhibit a wide range of therapeutic effects including antipsychotic, antiemetic, antihistaminergic and anticholinergic activities. Apart from these well-known effects, phenothiazines have also been evaluated for their anti-proliferative effect on various cell types as well as their ability to act synergistically with various chemotherapeutic agents [45].

The chemical structures of chlorpromazine and thioridazine, as examples of the general phenothiazine structure, are given in Fig. (2).

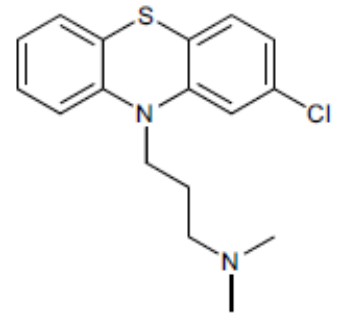

Chlorpromazine

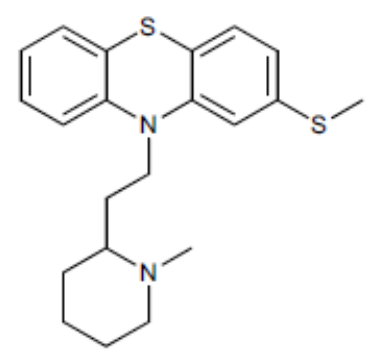

Thioridazine

Fig. (2). Phenothiazine chemical structures.

The polycyclic ring structure, which is important for the neurological action of the class, contributes to the lipophilicity of the compound and can be substituted with various groups on $C_{-2}$ as well as the thiazine nitrogen to yield derivatives with varying therapeutic, and side effect profiles. A number of side effects including dyskinesia and extrapyramidal effects are commonly associated with these compounds but, when the intended therapeutic effect is antibacterial or chemotherapeutic synergism, any effect 
on eukaryotic cells may become an unwanted effect. The structure activity relationships for antipsychotic, calmodulin inhibitory, anti-proliferative and P-glycoprotein inhibitory activity of the phenothiazines were reviewed by Jaszczyszyn and colleagues [45]. Phenothiazine-type compounds are often designated as piperazine, piperidine or aliphatic phenothiazines based on the nature of the thiazine nitrogen substitution. Piperazine phenothiazines exhibit the strongest neuroleptic action with electronegative substitutions on $C-2$ further increasing the antipsychotic activity. The length of the carbon linker between the phenothiazine ring and the terminal nitrogen moiety influences the affinity of these compounds for the dopamine receptor but has also been linked to the calmodulin inhibitory activity of the phenothiazines. Calmodulin inhibition may play a role in the anti-proliferative and P-glycoprotein (P-gp) inhibitory activity of this class [46]. The $C$-2 substitution on the phenothiazine core, the presence of the lipophilic aromatic ring and the terminal amine function also seems to play an important role in calmodulin inhibition. Phenothiazine derivatives are weak bases which are mostly protonated at physiological $\mathrm{pH}$. The amphiphilic nature of these compounds is important for their calmodulin inhibitory activity but may also play a role in their interaction with efflux pumps. The lipophilic cationic nature of the molecule also determines the degree of interaction with lipid membranes and thus influences the distribution patterns of the class [45-48].

Many authors are currently advocating for the inclusion of phenothiazines, more specifically thioridazine, in the treatment regimen for drug resistant tuberculosis (TB). Thioridazine has proven effective as an adjunct therapy in non-responsive MDR patients, and with careful management of its side effects, may become a valuable drug in the management of drug resistant TB [49-51]. Various therapeutically employed phenothiazines have been evaluated for antimycobacterial activity. Thioridazine and chlorpromazine demonstrate comparatively effective in vitro activity [52] but, as thioridazine presents a better side effect profile, most research efforts have focused on thioridazine and derivatives thereof [51]. It has been suggested that phenothiazines exert their activity through (1) enhancement of intracellular killing by macrophages, (2) direct antimycobacterial activity via various mechanisms as well as (3) modulation of the pathogen efflux activity thereby potentiating activity of co-administered antimycobacterials.

The mechanism of antimicrobial activity of the phenothiazines has been investigated by a number of researchers. Thioridazine may modulate mycobacterial cell envelope integrity as well as interact with enzymes and proteins involved in the electron transport chain and aerobic respiration of bacterial cells, thereby altering the energy metabolism of $M$. tuberculosis [53-55]. Antimicrobial activity may be due to one, or a combination of these actions and even though concentrations necessary for in vitro antimycobacterial activity is therapeutically unattainable, accumulation of phenothiazines in macrophages afford these compounds in vivo activity $[47,51,56]$. 
Basic lipophilic drugs are known to accumulate extensively in tissues. This tissue accumulation is attributed to binding to phospholipids and accumulation in acidic compartments like lysosomes. The physicochemical properties of molecules as well as the phospholipid pattern and lysosome distribution of tissues determine which of the abovementioned methods are mainly responsible for the tissue accumulation [47]. Phospholipid binding results from an interaction of the basic functional groups of the molecules with the outward facing acidic moieties of the phospholipid layer [57] whereas lysosomal trapping is a function of the $\mathrm{pH}$ difference between the cytosol and lysosome. Amine containing neutral or weakly basic molecules in their unionised form diffuse across lysosomal membranes and become ionised in the acidic lysosomal environment. Protonation of these compounds limits passive diffusion across the lysosomal membrane and accordingly results in accumulation of the compound within the lysosome [47, 48]. The lipophilicity and acid dissociation constant of the various functional groups of drugs prone to lysosomal trapping will influence the amount and rate of accumulation within the lysosome. Chlorpromazine $\left(\log \mathrm{P}=4.54\right.$, and $\mathrm{p} K_{\mathrm{a}}=9.2$ Chemaxon) and thioridazine ( $\log \mathrm{P}=5.47$, and $\mathrm{p} K_{\mathrm{a}}=8.93$ Chemaxon) are prone to lysosomal accumulation and accordingly concentrate in macrophages which are rich in lysosomes [47, 56]. Small changes in physicochemical properties or intracellular conditions may result in significant changes in the degree of lysosomal accumulation. It may also be worth noting that lysosomal accumulation is energy dependent (proton gradient between cytosol and lysosome is maintained by ATP-dependent proton pumps) and the extent of the trapping will likely be influenced by drug-drug interactions at a cellular distribution level [47, 48]. As the build-up of these compounds in macrophages plays an important part in achieving sufficient concentrations for in vivo antimycobacterial activity, functional and physicochemical properties that allow for this accumulation must be considered when phenothiazines are derivatized for the purpose of improving antimycobacterial activity.

Various reviews on the activity of phenothiazines in mycobacteria mention that, apart from their direct antimicrobial action, phenothiazines promote intracellular killing of $M$. tuberculosis in macrophages [3, 34, 51, 58-61]. Potentiation of intracellular killing is attributed to the ability of these compounds to modulate potassium and calcium transport to- and from the lysosome resulting in acidification of the lysosomal environment and subsequent killing of the bacteria [28, 34, 58]. Martins and colleagues demonstrated that various compounds that inhibit $\mathrm{K}^{+}$and $\mathrm{Ca}^{2+}$ movement across membranes (e.g. verapamil and reserpine) have the ability to promote intracellular killing of MDR tuberculosis by non-killing macrophages. The authors subsequently suggest that this approach could be explored as an alternative method of managing mycobacterial infections [28, 58]. Targeting eukaryotic $\mathrm{K}^{+}$and $\mathrm{Ca}^{2+}$ transport may result in host toxicity but accumulation of phenothiazines in macrophages affords these compounds a certain degree of selective toxicity as systemic concentrations could be maintained at concentrations below that which would result in unacceptable host side effects. The phenothiazine group may therefore be good lead 
compounds for drugs that promote intracellular killing of bacteria [58]. As noted above, properties that result in the accumulation of these compounds in macrophages should be maintained in the rational design of compounds that target this pathway.

In addition to the 2 antimycobacterial mechanisms described above, a number of studies report phenothiazine-mediated intracellular EB accumulation and antimycobacterial potentiation. These effects were attributed to possible efflux pump inhibitory activity by this group of compounds [28, 61, 62]. As mentioned previously, EB accumulation and potentiation of the activity or synergy observed with coadministered antimycobacterials may be due to effects other than efflux pump inhibition. A study by De Keijzer and colleagues quantitatively studied the proteome of $M$. tuberculosis and evaluated the accumulation of fluorescent dyes in cells of mycobacteria treated with thioridazine [55]. The authors suggest that increased cell wall permeability induced by thioridazine treatment may be the basis for the synergistic effect observed between thioridazine and various antimycobacterials. The study however also mentions that thioridazine may indirectly inhibit the activity of efflux pumps through reduction of energy available for active efflux as well as membrane mediated inhibition of efflux pumps resulting from the effect of thioridazine on mycobacterial cells. These mechanisms for efflux pump inhibition were also suggested by other authors [59, 61]. There have thus far not been any reports of a direct interaction of the phenothiazine-type compounds with mycobacterial efflux pumps and where these compounds inhibit efflux, it is likely through indirect modulation of efflux pump activity.

A recent study by Pieroni and colleagues used a ligand-based approach to design and synthesize thioridazine derivatives for the purpose of obtaining a compound with improved efficacy as an antimycobacterial adjuvant while limiting the side effects observed with thioridazine [23]. The nature of the heterocyclic ring was adapted to minimize neurological side effects while the $N$-methylpiperidine moiety was maintained, likely in consideration of efflux pump substrate affinity as well as basic amine related protonation in acidic lysosomes. The four derivatives that were selected for screening on $M$. tuberculosis all demonstrated reduced direct antimycobacterial activity, reduced toxicity towards human macrophages, good synergistic activity with various first and second line antimycobacterials in vitro, and potentiated the effect of rifampicin and isoniazid ex vivo at concentrations that were shown to be nontoxic to macrophages. The study also measured the effect of the synthesized compounds on EB accumulation and ultimately suggests efflux pump inhibitory activity as a mechanism for observed potentiation of antimycobacterials. This study is unique as it is the first report of rational design efforts aimed at the improvement of thioridazine for the purpose of enhancing its potential specifically as adjuvant antimycobacterial therapy. Positive results from this study show that with careful consideration of molecular properties required for activity and cellular distribution, it may be possible to use a ligand-based approach for the rational development of adjuvants for TB therapy, 
even in the absence of sufficient information relating to target or mechanism of action of the compounds.

\section{Piperine}

Piperine is the primary alkaloid responsible for the pungency of black pepper. The experienced pungency results from activation of highly calcium selective, vanilloidtype transient receptor potential cation channels (TRPV) [63]. Piperine is a piperidine derivative linked to a benzodioxole moiety via a 5 -carbon linker. The ketone adjacent to the basic nitrogen of the piperidine moiety influences the protonation of the tertiary piperidine nitrogen at physiological $\mathrm{pH}$. It has a predicted $\log \mathrm{P}$ of 2.78 (Chemaxon) and is poorly soluble above $100 \mu \mathrm{g} / \mathrm{ml}$, complicating its evaluation on the growth of microbes above this concentration [30].

A plethora of therapeutic activities have been described for piperine and its derivatives $[64,65]$. Amongst others, piperine non-competitively inhibits Pglycoprotein activity and is able to reduce the MIC of rifampicin and inhibit intracellular growth of $M$. tuberculosis [26, 30]. Piperine is reported to enhance the bioavailability of several unrelated pharmaceuticals and supplements through interference with drug absorption and metabolism [66]. The structure activity relationships for a number of these activities were recently reviewed [67]. Of particular interest to this review are the SAR for potentiation of ciprofloxacin activity (attributed to inhibition of the NorA efflux pump inhibitory activity) [26, 68,69] and SAR for modulation of CYP450 enzyme activity. The NorA efflux pump is a MFS-type efflux pump in Staphylococcus aureus and as piperine has been shown to modulate both NorA and Rv1258c efflux activity [26], it may be worth considering SAR for NorA EPI when designing piperidine derivatives aimed at EP inhibition in mycobacteria. SAR evaluations for NorA inhibitory activity have shown that the amide carbonyl is important for EP inhibition. The piperidine ring can however be replaced by various moieties on the amide nitrogen to enhance EPI activity. Substitutions containing electron withdrawing groups in this position also potentiate efflux inhibition. An unsaturated aliphatic chain seems to be important for EP inhibitory activity and the addition of alkyl chains, particularly ethyl and n-propyl, on C-4 further increases potency. The benzodioxole moiety was found to contribute to the NorA EP inhibition of these compounds [67].

Docking studies performed by Sharma and colleagues used a predicted $3 \mathrm{D}$ model of Rv1258c to model the interaction of piperine with this MSF-type mycobacterial efflux pump [26]. Docking predictions indicated that the ketone function in piperine may form a hydrogen bond with an arginine moiety within the suggested active site of the Rv1258c model. Several other interactions were also observed with various residues within this binding pocket. The authors suggest that the abovementioned hydrogen bond may contribute to the efflux pump inhibitory activity of piperine. As discussed previously, Singh and colleagues also used a model of Rv1258c to perform docking studies of reserpine, piperine, verapamil and verapamil analogues [27]. Apart from the 
verapamil binding interactions observed, their docking predictions also indicated possible hydrogen bond formation of the oxygen functions of the benzodioxole moiety of piperine with an arginine and glycine moiety in the putative binding site of Rv1258c. The authors suggest that hydrogen bonds may stabilize these proteininhibitor complexes.

Unfortunately, the ability of piperine to interact with P-glycoprotein [66] and CYP450 enzymes will likely result in indiscriminate drug-drug interactions with coadministered medication and is certain to complicate the use of piperine-type compounds as boosters of antimycobacterial activity. SAR evaluations for enzyme inhibitory activity have shown that the specific combination of the methylenedioxyphenyl ring, alkyl linker and basic piperine moiety plays an important role in enzyme inhibition. Altering one or a combination of these moieties influences enzyme inhibitory activity but can also alter selectivity towards either inducible or constitutive enzymes specifically. A detailed overview of SAR for rat hepatic microsomal constitutive and inducible cytochrome $\mathrm{P} 450$ enzyme activity is described in a paper by Koul and colleagues [70] and these activities could be considered to potentially minimize enzyme inhibition.

Thus, taking the above into consideration, rational drug design endeavours targeting selective prokaryotic efflux pump inhibitory activity of piperine derivatives may consider maintaining the hydrophobic portion of the molecule but also including various hydrogen bond acceptors in order to allow stabilization of the interaction of the molecule with the efflux pump. Excluding structural features that potentiate enzyme interactions but which are not essential for EPI activity may reduce the side effect profile and drug-drug interaction of derivatives.

\section{Other structures of interest}

Although limited data exists around the M. tuberculosis EPI activity and mechanism of potentiation of other antimycobacterials for the compounds discussed below, the potential for development of these structures into future EPIs and/or structural and bioactivity similarities with well described is EPIs warrant a brief mention in this review.

\subsection{Berberine}

Berberine (Fig. 3) shares a number of structural features with piperine and is an amphipathic, naturally occurring iso-quinoline-type alkaloid with antimicrobial properties. It is a well-recognized efflux pump substrate and it has been demonstrated that the intracellular concentration of berberine is increased, and antimicrobial effect potentiated, when it is co-administered with efflux pump inhibitors [71, 72]. Berberine however similarly, potentiates the effect of several classes of co-administered antibiotics $[73,74]$. Potentiation of antibiotic effect may result from competitive inhibition of efflux. Direct antimycobacterial activity has also been reported for berberine and derivatives [75] thereby adding to the overall value of including these compounds as 
part of an antimycobacterial regimen. Oral bioavailability of berberine may however be problematic and the reported low plasma levels after oral administration is attributed to, amongst others, poor absorption from the GIT, extensive first pass metabolism in the intestine and P-glycoprotein mediated efflux [76].
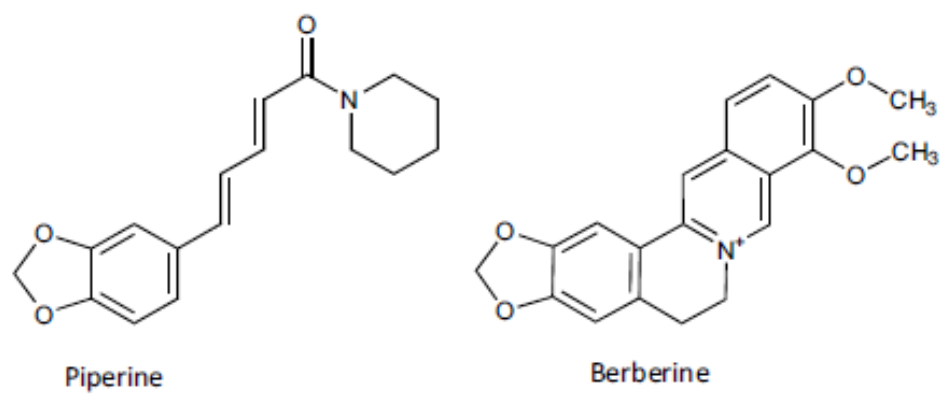

Fig. (3). Piperine and berberine.

\subsection{Tetrandrine}

The natural bis-benzylisoquinoline alkaloid, L-type calcium channel and Pglycoprotein inhibitor, tetrandrine (Fig. 4) was able to reduce the MIC of isoniazid and ethambutol in clinical $M$. tuberculosis strains [77]. Similar to verapamil, tetrandrine contains hydrophobic planar aromatic rings as well as tertiary nitrogens of which at least one will likely be protonated at physiological $\mathrm{pH}$. These moieties have been identified as important structural features of P-gp mediated MDR reversing agents [77]. Tetrandrine may therefore modulate mycobacterial efflux pump activity via a similar mechanism as verapamil.

\subsection{Reserpine}

Reserpine is classified as an indole-type alkaloid with antipsychotic and antihypertensive properties. It is however rarely used therapeutically due to its extensive side effect profile. Reserpine binds irreversibly to the vesicular mono-amine transporter membrane protein to block the transport of monoamine neurotransmitters [78]. Calcium channel antagonism has also been described for this compound [79]. Reserpine is able to inhibit efflux mediated by a variety of classes of efflux pumps and various studies have demonstrated that reserpine is able to reduce the MIC of- or increase mycobacterial susceptibility to a number of antimycobacterials [80-84]. It has been reported that reserpine mediates efflux via an interaction with glycine residues within the MFS-type, Bacillus subtilis efflux pump (Bmr) [85]. 

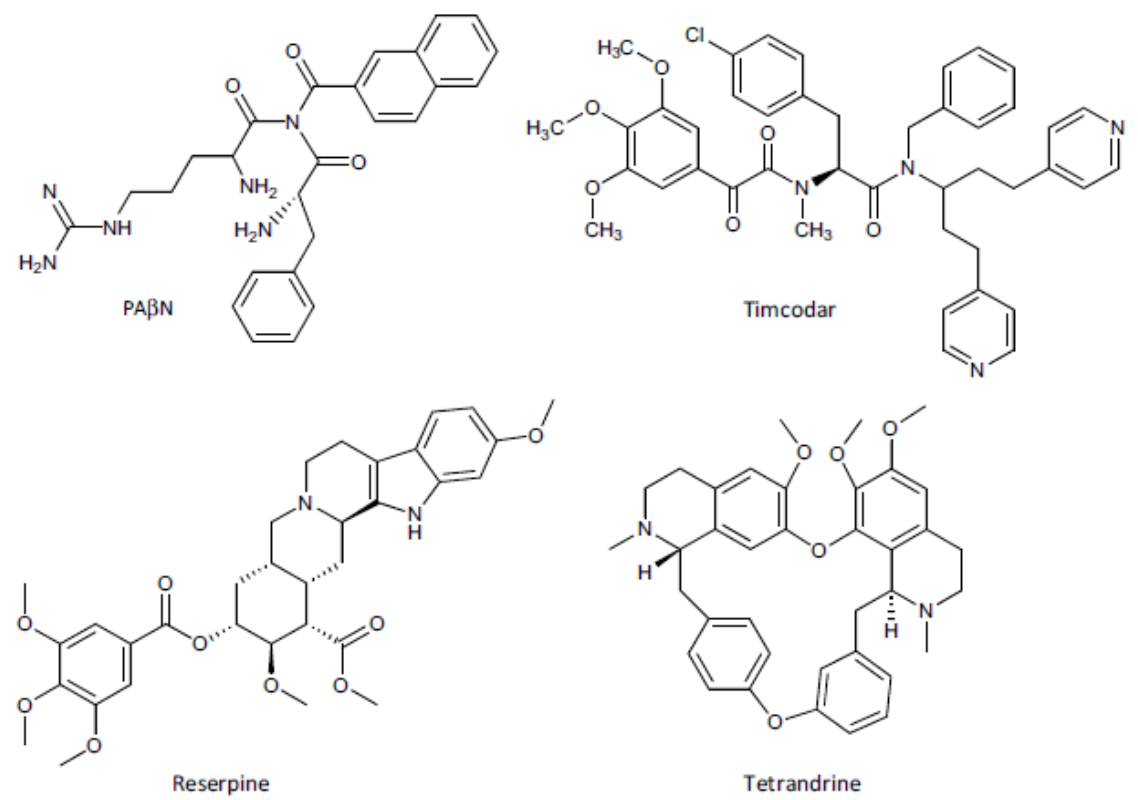

Fig. (4). Structures of interest for EPI activity.

\subsection{Timcodar}

It was recently demonstrated that timcodar is able to increase the potency of rifampicin, bedaquiline and clofazimine in vitro, ex vivo and in vitro. The authors suggest that this potentiation likely results from a combination of bacterial and hosttargeted mechanisms [86]. Timcodar and biricodar are $3^{\text {rd }}$ generation modulators of Pgp and multidrug resistance proteins (MRPs) evaluated as enhancers of cancer therapy $[87,88]$. Timcodar is highly lipophilic ( $\log \mathrm{P}=6.87$ chemaxon) with none of the tertiary nitrogens likely to be protonated at physiological $\mathrm{pH}$. Despite biricodar entering phase II clinical trials in 1998, neither of these compounds has received FDA approval as adjunctive therapy in cancer chemotherapy.

\subsection{PAßN}

Phenylalanyl arginyl $\beta$-naphthylamide $(\mathrm{PA} \beta \mathrm{N})$ is a peptidomimetic EPI primarily reviewed for its activity on RND-type efflux pumps. The compound contains a guanidine moiety which will likely be protonated, as well as a number of primary amines which may be protonated to some extent at physiological $\mathrm{pH}$. The development of PA $\beta \mathrm{N}$ and derivatives into therapeutic agents has been complicated by pharmacokinetic and toxicity problems experienced with this class. PA $\mathrm{N}$ was identified as a lead compound from a large-scale screening of small molecules and a number of subsequent derivatives were made with the goal of improving activity, serum stability and toxicity profiles of the group [25, 89]. Compared to verapamil and thioridazine, PA $\beta \mathrm{N}$ has not been as extensively evaluated for mycobacterial EPI activity. Balganesh and colleagues constructed $M$. tuberculosis knockout (KO) mutants of a number of efflux pumps from various efflux pump families and demonstrated that, when incubated with PA $\beta \mathrm{N}$ over the course of a few days, it was able to reduce the MIC of a pyrazolone-type compounds in the wild type but also to a lesser extent the KO 
mutants [90]. Their results indicate that the MIC reduction brought on by PA $\beta \mathrm{N}$ is likely due to inhibition of efflux activity. The ability of PA $\beta \mathrm{N}$ to still influence the MIC in the various $\mathrm{KO}$ mutants may however point to indiscriminate inhibition of a number of efflux pumps and/or contribution of an additional potentiation mechanism. After recent reports of outer membrane permeabilizing activity by PA $\beta \mathrm{N}$ [91], a study by Misra and colleagues employed real-time efflux assays to distinguish between efflux inhibition and membrane activity. The study concluded that the main mechanism of action of PA $\mathrm{N}$ is inhibition of efflux activity followed by a delayed and weaker membrane destabilizing action likely observed after prolonged incubation of cells with $\mathrm{PA} \beta \mathrm{N}$. The authors do however mention that the cell wall permeabilizing activity of PA $\beta N$ may be amplified in cells not sufficiently expressing efflux pumps [92]. These results may shed some light on the activity of PA $\beta \mathrm{N}$ as observed on knockout mutants of efflux pumps from various EP families as observed by Balganesh [90]. PA $\beta \mathrm{N}$ and derivatives are believed to competitively inhibit efflux of several substrates of RNDtype efflux pumps to varying degrees depending on their exact binding pocket in the transporter protein. A comprehensive review on the mechanism of efflux inhibition of RND-type efflux pumps describes efforts to maximize activity and reduce toxicity of this class of compounds in gram negative bacteria [25]. The basic moieties on the structure that were shown to be important for efflux inhibitory activity of this class also afford unfavourable pharmacokinetic properties and increased toxicity to these compounds and $\mathrm{PA} \beta \mathrm{N}$ is therefore primarily used in efflux assays with little prospect of being developed into a therapeutic agent $[25,89]$.

\subsection{Spectinamides}

Spectinomycin (Fig. 5) is an antibiotic with structural similarities to the aminoglycoside antibiotics. The 1,3-diaminoinositol pharmacophore is uniquely linked to a sugar moiety to form tricyclic-type structure. It demonstrates strong bacterial ribosomal affinity with resulting inhibition of bacterial protein synthesis. It binds selectively to the bacterial ribosomal subunit and therefore does share the toxicity profile of the standard aminoglycoside antibiotics. The unexpectedly low antimycobacterial effect of spectinomycin is attributed to extensive efflux by the Rv1258c efflux pump [16]. Lee and colleagues were able to design spectinomycin analogues that are able to circumvent active efflux by substituting the ketone function on the sugar with various amide carbonyl-linked functional groups. Pyrrole and piperidine rings, particularly halogen substituted piperidine analogues seem to demonstrate the most promising antimycobacterial and efflux pump circumventory activity [16]. These spectinamide derivatives demonstrate good affinity and selectivity for the bacterial ribosome with significantly improved antimycobacterial activity compared to the original spectinomycin molecule. Subsequent research by this group established that the lead spectinamide derivative 1599 (Fig. 5) is able to reduce the MIC of various co-administered antimycobacterials [93]. The researchers observed that co-administration of 1599 did not potentiate the activity of spectinomycin. This would suggest that the ability of 1599 to circumvent EP-mediated resistance is not due to 
inhibition of Rv1258c efflux as EP inhibition would have resulted in improved activity of spectinomycin when co-administered with 1599.
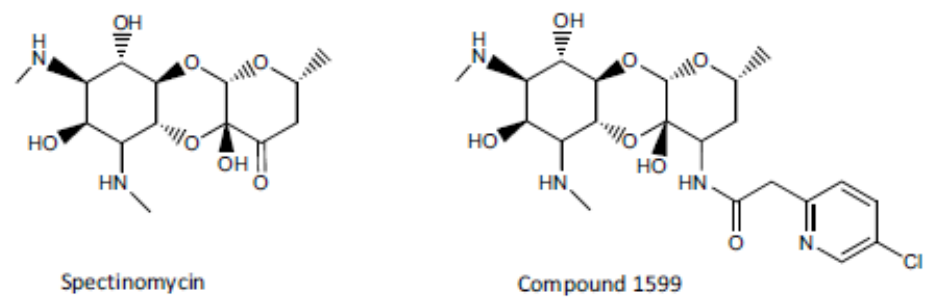

Fig. (5). Spectinomycin and spectinamide derivatives.

\section{P-plycoprotein transport inhibitors}

P-gps are well characterized transmembrane ATP-type transporter pumps that modulate transport of a large variety of substrates in the intestine, liver, kidneys and across the blood brain barrier amongst others [94]. P-gp transporter pumps influence the distribution and toxicity profiles of a broad range of compounds and their overexpression in certain cancer cells made modulation of these, and other similar pumps attractive targets to improve the efficacy of chemotherapy. Various P-gp inhibitors, of which verapamil was one of the first, were characterised and developed in an attempt to modulate the effect of efflux through these transporter pumps. The wide distribution and large range of physiological functions of P-gp pumps as well as the non-specific substrate recognition observed with these efflux proteins, however resulted in unpredictable effects and numerous drug-drug interactions. To better predict the likelihood of inhibition of P-gp pumps and aid rational drug development of more specific P-gp inhibitors, various structure activity relationship studies were undertaken, and numerous models developed to predict substrates and inhibitors of this efflux pump type.

In silico prediction of substrates and inhibitors of $\mathrm{P}$-gp activity is complicated by the promiscuous substrate recognition as well as a multifaceted molecule-transporter pump interaction profile which could include competitive as well as non-competitive inhibition or modulation [95]. A recent article by Montenari and collegues reviewed current advances in prediction of ABC-transporter substrate and inhibitor interactions [96]. The authors report that structure activity relationships for P-gp interaction have been described for a range of diverse molecular scaffolds and suggest that this may point to local effects or binding sites unique to the type of scaffold within the P-gp pump. The suggested structure activity relationships often relate to general physicochemical properties of the molecule which may include lipophilicity, the presence of aromatic rings, functional groups that may become charged and the ability of the molecule to form hydrogen bonds. The authors also state that a clear set of rules for the molecular basis for molecule-pump interaction is yet to be established and that there is currently no definitive guide to aid the rational development of P-gp inhibitors or modulators [96]. 
Research published by Broccatelli and colleagues in 2011 [97] demonstrated the importance of considering both specific (related to the pharmacophore) and nonspecific (general physicochemical/ADME) descriptors when predicting P-gp inhibition. The authors also note that molecules which may non-competitively inhibit P-gp efflux for example flavonoid and steroid type molecules require special consideration when attempting to predict P-gp inhibition in silico. Through a combination of model types that consider specific as well as general properties, the authors were able to predict P-gp inhibition with a good degree of accuracy. For competitive inhibition, the size, shape and flexibility of the molecule, the presence of a large hydrophobic area and at least one hydrogen bond acceptor as well as Log P of the molecule, seem to be strong predictors of inhibitory activity. The authors noted that a $\log \mathrm{P}$ of less than 2 was a strong indicator of a non-inhibitor. Similarly, the role of nitrogens able to accept or donate hydrogens depending on the environment, was also highlighted as an important consideration of P-gp activity. For non-competitive inhibition (e.g. flavonoid and steroid-type molecules), size and flexibility seem to play a lesser role in predicting activity [97].

P-gp activity would most likely be an undesirable trait for efflux pump inhibitors targeting bacterial efflux pumps and it would be pertinent to consider, and where possible minimise, molecular properties that increase the likelihood of P-gp modulation. Due to the large variety of substrate and inhibitor scaffolds as well as the variable binding sites within the P-gp pump, the guidelines for predicting P-gp interaction are relatively vague. Consideration of the specific chemical scaffold as well as whether competitive or non-competitive (e.g. piperine) inhibition would be more likely for the structure under investigation would allow the design of a molecule that might be less likely to interact with P-gp pumps.

\section{Conclusion}

Ever increasing levels of drug resistance in $M$. tuberculosis necessitates novel approaches in utilization of available treatment options as well as in the design of future antimycobacterials. Apart from effectively targeting the pathogen, approaches to drug therapy of $M$. tuberculosis should centre around 1) preventing development of resistance, 2) overcoming current resistance and 3) developing future antimycobacterials that are less prone to the development of resistance.

Efflux pump activity in $M$. tuberculosis contribute to low level antimycobacterial resistance as well as the development of high level resistance through reduction of intracellular antimycobacterial concentrations. Inhibition of efflux activity is therefore an attractive target to overcome and prevent development of resistance to antimycobacterials. Various Mycobacterial EPIs are described in literature. Therapeutic use of these EPIs are however hampered by their side effect profile and unpredictable drug-drug interactions resulting from inhibition of mammalian transporter pumps and ion channels, amongst others. 
Rational drug design endeavours have the potential to yield safer therapeutic EPIs with improved pharmacokinetic and pharmacodynamic properties. Knowledge relating to the mechanism of action and molecular target of the inhibition of efflux is however central to the rational drug design approach. Surprisingly little is known about the target site or even mechanism of action of EPIs in mycobacteria. The aim of this review was therefore to examine what is known about the molecular properties and mechanism of action of a range of compounds that have been identified as putative EPIs in $M$. tuberculosis and highlight key considerations for the design of compounds for the purpose of minimizing efflux of antimycobacterials.

From the literature it is evident that the term efflux pump inhibitor is not necessarily indicative of a compound that directly binds to an efflux pump in order to inhibit efflux. Most studies investigating efflux pump inhibitory activity measure one or a combination of factors that may result from efflux pump inhibition by the compound under investigation. As a matter of fact, binding to efflux pumps is rarely demonstrated. The lack of crystal structures for efflux proteins in $M$. tuberculosis hampers in-depth investigation into possible binding of EPIs to efflux pumps and researchers have thus far resorted to homology modelling to predict binding interactions of a handful of putative EPIs.

Apart from biological inhibition of efflux pump expression, efflux can be inhibited by direct interaction with the efflux pump or through interference with the energy source required for active efflux. Depending on the efflux pump family, efflux pumps rely on PMF or ATP-based energy to effect active efflux. Any compound that interferes with the availability or effective utilization of cellular energy could thus potentially inhibit efflux pump activity. In fact, interference with energy availability is a popular theory for themechanism of efflux inhibition for various classes of EPIs. Not only protonophores, but any compound that interferes with the electrical of chemical gradient across the bacterial cell wall has the potential to reduce energy available for cellular functions. Amongst these are any compounds that interfere with the movement of ions across cell membranes for example verapamil and other calcium channel blockers as well as phenothiazine-type compounds. There seems to be consensus amongst authors that the phenothiazine class effects efflux inhibition through mechanisms other than a direct interaction with efflux pumps. Limited information and varied opinions however exist regarding the mechanism of efflux inhibition for other EPIs. It is possible that certain EPIs could inhibit efflux via an energy dependent or direct binding mechanisms, or a combination thereof, depending on assay conditions. It is clear from this review that there is limited information available relating to the mechanism of action of any of the classes of putative EPIs in mycobacteria. Rational drug design efforts will therefore have to draw on information available for a particular type of EPI in other cell types, explore ligand-based drug design routes and consider employing homology models of the target efflux pump to 
predict binding interactions and thereby molecular requirements to maximise ligandprotein interactions.

In addition to good efflux inhibition, selective toxicity of EPIs should be a key consideration in the rational design of therapeutically safe EPIs. Achieving sufficient selective toxicity with compounds that afford efflux inhibition via a reduction in cellular energy may be difficult due to similarities in energy generation and utilization across cell types. Lysosomal accumulation of the phenothiazine-type compounds allows for the use of this class despite host side effects as accumulation in macrophages allow for the use of much lower doses than would normally be required to affect antimycobacterial activity. Any compounds designed to target the availability of energy for active efflux should accordingly prioritize selectivity over activity if compounds are to be used therapeutically. Similarly, where a direct interaction with efflux pumps is targeted, this interaction should be selective towards prokaryotic efflux pumps. Interactions with the P-gp efflux pumps for example, will complicate use of EPIs as these interactions are likely to result in drug-drug interactions and unpredictable plasma levels of any co-administered medication. P-gp inhibitory activity is relatively common with putative bacterial EPIs and has been described for verapamil, piperine, timcodar, tetrandrine and reserpine amongst others. This is not surprising as there seems to be a good degree of overlap between molecular properties that increase recognition by bacterial as well as mammalian efflux pumps. These characteristics include a higher Log P, distinct hydrophobic and hydrophilic areas within the molecule, the presence of atoms like basic nitrogens which may become charged and the ability of the molecule to form hydrogen bonds. In addition to P-gp inhibitory activity, a tendency to modulate mammalian ion channel activity also seem to be a common trend across numerous classes of compounds for which prokaryotic EPI activity has been described. Verapamil, tetrandrine, reserpine and the phenothiazine class compounds, amongst others, all demonstrate ion channel inhibitory activity. The ability of these compounds to block efflux pumps may however not necessarily be linked to their ability to block ion channels as is observed with $R$-verapamil and norverapamil which are able to maintain their capacity to reverse drug tolerance despite their reduced cardiac activity when compared to verapamil. In order to minimize side effects and reduce drug-drug interactions, molecular properties that potentiate interactions with host transporter pumps, ion channels and enzyme systems should be considered throughout the drug design process.

Efflux pumps likely recognise potential substrates based on their physicochemical properties in addition to their molecular structure. This likely contributes to the indiscriminate efflux of a range of structurally diverse compounds by a single efflux pump type. To afford a compound efflux antagonism via competitive interaction with an efflux pump, the potential antagonist would likely have to share similar features to efflux pump substrates in order to gain entry or get into close proximity to the substrate binding pocket within the efflux protein. Once access to the binding pocket 
is attained, binding interactions with residues within the pocket should be of a more permanent nature than the substrate if anything more than purely competitive inhibition is to be achieved. The presence of moieties which are able to form hydrogen bonds may increase binding affinity of the putative EPI. Research suggests that efflux pumps preferentially transport compounds with clear hydrophilic and lipophilic regions and in a similar fashion, many putative EPIs also share this amphiphilic nature. Verapamil with its tertiary amine which is protonated at physiological $\mathrm{pH}$ is an example of this. It may therefore be useful to examine the molecular properties of known efflux pump substrates when embarking on rational drug design of an efflux pump inhibitor targeting a particular efflux pump(s). This approach may however not apply to compounds which act as non-competitive inhibitors of efflux.

The strategies mentioned above describe the rational development of EPIs that would likely be used in combination with an antibiotic which is a substrate for efflux pump(s). An alternative approach to employing EPIs in a polypharmacy approach is to design new, or adapt current antimycobacterials to naturally circumvent efflux. This was achieved with the spectinomycin antibiotics through altering the physico-chemical properties that increase the likelihood of compounds being recognized as efflux pump substrates. Alternatively, strategies that increase cell wall permeation of compounds also has the potential to increase the intracellular concentration of antimycobacterials to concentrations above the extrusion capacity of efflux pumps. The addition of bulky polycyclic moieties to current or new antimycobacterials may reduce the effect of active efflux by decreasing the substrate fit within the efflux pump as well as through increased penetration into the mycobacterial cell by means of the increased lipophilicity afforded by the addition of these lipophilic moieties. Hybrid compounds as an alternate to the polypharmacy approach may also have several, particularly pharmacokinetic, advantages over co-administration of EPIs with antimycobacterials.

Circumvention of efflux-based resistance remains an attractive strategy to improve the therapeutic management of mycobacterial disease. Even though this field has been explored for quite some time, the side effect profiles and unpredictable drug-drug interactions observed with possible EPI candidates has thus far precluded the therapeutic use of these compounds. Rational drug design may offer a solution to this problem through consideration of molecular properties that may increase selectivity of drug candidates for bacterial efflux pumps or afford selective toxicity through various other mechanisms. With limited information available regarding the mechanism of action and/or binding site of EPIs, the medicinal chemist will have to draw information from various sources and employ a combination of strategies to enable rational drug design of therapeutic EPIs. 


\section{Consent for publication}

Not applicable.

\section{Conflict of interest}

The authors declare no conflict of interest, financial or otherwise.

\section{Acknowledgements}

We are grateful to the National Research Foundation of South Africa for financial support. SLS is funded by the South African Research Chairs Initiative of the Department of Science and Technology and National Research Foundation (NRF) of South Africa, award number UID 86539. The content is solely the responsibility of the authors and does not necessarily represent the official views of the NRF. 


\section{References}

[1] da Silva, P.E.; Von Groll, A.; Martin, A.; Palomino, J.C. Efflux as a mechanism for drug resistance in Mycobacterium tuberculosis. FEMS Immunol. Med. Microbiol., 2011, 63, 1-9.

[2] Sarathy, J.P.; Dartois, V.; Lee, E.J.D. The role of transport mechanisms in mycobacterium tuberculosis drug resistance and tolerance. Pharmaceuticals (Basel), 2012, $5,1210-1235$.

[3] Viveiros, M.; Martins, M.; Rodrigues, L.; Machado, D.; Couto, I.; Ainsa, J.; Amaral, L. Inhibitors of mycobacterial efflux pumps as potential boosters for anti-tubercular drugs. Expert Rev. Anti Infect. Ther., 2012, 10, 983-998.

[4] Bottger, E. C. In Drug resistance in Mycobacterium tuberculosis: Molecular mechanisms and susceptibility testing; Donald, P. R., Van Helden, P. D., Eds.; Antituberculosis Chemotherapy; Karger Medicinal and Scientific Publishers: Basel, 2011, pp. 128.

[5] Marquez, B. Bacterial efflux systems and efflux pumps inhibitors. Biochimie, 2005, 87, 1137-1147.

[6] Louw, G.E.; Warren, R.M.; Gey van Pittius, N.C.; McEvoy, C.R.; Van Helden, P.D.; Victor, T.C.A balancing act: efflux/influx in mycobacterial drug resistance. Antimicrob. Agents Chemother., 2009, 53, 3181-3189.

[7] De Rossi, E.; Ainsa, J.A.; Riccardi, G. Role of mycobacterial efflux transporters in drug resistance: an unresolved question. FEMS Microbiol. Rev., 2006, 30, 36-52.

[8] Black, P.A.; Warren, R.M.; Louw, G.E.; van Helden, P.D.; Victor, T.C.; Kana, B.D. Energy Metabolism and Drug Efflux in Mycobacterium tuberculosis. Antimicrob. Agents Chemother., 2014, 58, 2491-2503.

[9] Putman, M.; van Veen, H.W.; Konings, W.N. Molecular properties of bacterial multidrug transporters. Microbiol. Mol. Biol. Rev., 2000, 64, 672-693.

[10] Van Bambeke, F.; Pages, J.M.; Lee, V.J. Inhibitors of bacterial efflux pumps as adjuvants in antibiotic treatments and diagnostic tools for detection of resistance by efflux. Recent. Pat. Antiinfect Drug Discov., 2006, 1, 157-175.

[11] Dinesh, N.; Sharma, S.; Balganesh, M. Involvement of Efflux Pumps in the Resistance to Peptidoglycan Synthesis Inhibitors in Mycobacterium tuberculosis. Antimicrob. Agents Chemother., 2013, 57, 1941-1943.

[12] Kourtesi, C.; Ball, A.R.; Huang, Y.Y.; Jachak, S.M.; Vera, D.M.; Khondkar, P.; Gibbons, S.; Hamblin, M.R.; Tegos, G.P. Microbial efflux systems and inhibitors: approaches to drug discovery and the challenge of clinical implementation. Open Microbiol. J., 2013, 7, 34-52. [13] Van Bambeke, F.; Michot, J.M.; Tulkens, P.M. Antibiotic efflux pumps in eukaryotic cells: occurrence and impact on antibiotic cellular pharmacokinetics, pharmacodynamics and toxicodynamics. J. Antimicrob. Chemother., 2oo3, 51, 1067-1077.

[14] Roy, K., Ed.; In Quantitative structure-activity relationships in drug design, predictive toxicology, and risk assessment; Medical Information Science: Hershey PA, USA, 2015.

[15] Viveiros, M.; Pieroni, M. Spectinamides: a challenge, a proof, and a suggestion. Trends Microbiol., 2014, 22, 170-171. 
[16] Lee, R.E.; Hurdle, J.G.; Liu, J.; Bruhn, D.F.; Matt, T.; Scherman, M.S.; Vaddady, P.K.; Zheng, Z.; Qi, J.; Akbergenov, R.; Das, S.; Madhura, D.B.; Rathi, C.; Trivedi, A.; Villellas, C.; Lee, R.B.; Rakesh; Waidyarachchi, S.L.; Sun, D.; McNeil, M.R.; Ainsa, J.A.; Boshoff, H.I.; Gonzalez-Juarrero, M.; Meibohm, B.; Bottger, E.C.; Lenaerts, A.J. Spectinamides: a new class of semisynthetic antituberculosis agents that overcome native drug efflux. Nat. Med., 2014, 2O, 152-158.

[17] Pagès, J.; Amaral, L. Mechanisms of drug efflux and strategies to combat them: Challenging the efflux pump of Gram-negative bacteria. Biochimica et Biophysica Acta (BBA) - Proteins Proteom., 2009, 1794, 826-833.

[18] Zechini, B.; Versace, I. Inhibitors of multidrug resistant efflux systems in bacteria. Recent. Pat. Antiinfect. Drug Discov., 2009, 4, 37-50.

[19] Bhardwaj, A.K.; Mohanty, P. Bacterial efflux pumps involved in multidrug resistance and their inhibitors: rejuvinating the antimicrobial chemotherapy. Rec. Pat. Antiinfect. Drug Discov., 2012, 7, 73-89.

[20] Amaral, L.; Martins, A.; Spengler, G.; Molnar, J. Efflux pumps of Gram-negative bacteria: what they do, how they do it, with what and how to deal with them. Front. Pharmacol., 2013, 4, 168.

[21] Tegos, G.P.; Haynes, M.; Strouse, J.J.; Khan, M.M.; Bologa, C.G.; Oprea, T.I.; Sklar, L.A. Microbial efflux pump inhibition: tactics and strategies. Curr. Pharm. Des., 2011, 17, 1291-1302.

[22] Martins, A.; Machado, L.; Costa, S.; Cerca, P.; Spengler, G.; Viveiros, M.; Amaral, L. Role of calcium in the efflux system of Escherichia coli. Int. J. Antimicrob. Agents, 2o11, $37,410-414$.

[23] Pieroni, M.; Machado, D.; Azzali, E.; Santos Costa, S.; Couto, I.; Costantino, G.; Viveiros, M. Rational design and synthesis of thioridazine analogues as enhancers of the antituberculosis therapy. J. Med. Chem., 2015, 58, 5842-5853.

[24] Te Brake, L.H.; Russel, F.G.; van den Heuvel, J.J.; de Knegt, G.J.; de Steenwinkel, J.E.; Burger, D.M.; Aarnoutse, R.E.; Koenderink, J.B. Inhibitory potential of tuberculosis drugs on ATP-binding cassette drug transporters. Tuberculosis (Edinb), 2016, 96, 150-157. [25] Opperman, T.J.; Nguyen, S.T. Recent advances toward a molecular mechanism of efflux pump inhibition. Front. Microbiol., 2015, 6, 421.

[26] Sharma, S.; Kumar, M.; Sharma, S.; Nargotra, A.; Koul, S.; Khan, I.A. Piperine as an inhibitor of Rv1258c, a putative multidrug efflux pump of Mycobacterium tuberculosis. $J$. Antimicrob. Chemother., 2010, 65, 1694-1701.

[27] Singh, K.; Kumar, M.; Pavadai, E.; Naran, K.; Warner, D.F.; Ruminski, P. G.; Chibale, K. Synthesis of new verapamil analogues and their evaluation in combination with rifampicin against Mycobacterium tuberculosis and molecular docking studies in the binding site of efflux protein Rv1258c. Bioorg. Med. Chem. Lett., 2014, 24, 2985-2990.

[28] Martins, M.; Viveiros, M.; Amaral, L. Inhibitors of $\mathrm{Ca}^{2+}$ and $\mathrm{K}+$ transport enhance intracellular killing of M. tuberculosis by non-killing macrophages. In Vivo, 20o8, 22, 6975 .

[29] Rodrigues, L.; Machado, D.; Couto, I.; Amaral, L.; Viveiros, M. Contribution of efflux activity to isoniazid resistance in the Mycobacterium tuberculosis complex. Infect. Genet. Evolut., 2012, 12, 695-700. 
[30] Adams, K.N.; Szumowski, J.D.; Ramakrishnan, L. Verapamil, and Its metabolite norverapamil, inhibit macrophage-induced, bacterial efflux pump-mediated tolerance to multiple anti-tubercular drugs. J. Infect. Dis., 2014, 21O, 456-466.

[31] Hille, B. Ion Channels of Excitable Membranes; Sinauer Associates inc: Sunderland, Massachusetts, 2001.

[32] Wright, S.H. Generation of resting membrane potential. Adv. Physiol. Educ., 2004, 28, 139-142.

[33] Golan, D.E., Ed.; In Principles of Pharmacology The Pathophysiologic Basis of Drug Therapy; Lippincott Williams \& Wilkins: Philadelphia, 2012.

[34] Amaral, L.; Martins, M.; Viveiros, M. Enhanced killing of intracellular multidrug resistant Mycobacterium tuberculosis by compounds that affect the activity of efflux pumps. J. Antimicrob. Chemother., 2007, 59, 1237-1246.

[35] Machado, D.; Pires, D.; Perdigao, J.; Couto, I.; Portugal, I.; Martins, M.; Amaral, L.; Anes, E.; Viveiros, M. Ion Channel Blockers as Antimicrobial Agents, Efflux Inhibitors, and Enhancers of Macrophage Killing Activity against Drug Resistant Mycobacterium tuberculosis. PLoS One, 2016, 11, e0149326.

[36] Farha, M.; Verschoor, C.; Bowdish, D.; Brown, E. Collapsing the Proton Motive Force to Identify Synergistic Combinations against Staphylococcus aureus. Chem. Biol., 2013, 2O, 1168-1178.

[37] Adams, K.; Takaki, K.; Connolly, L.; Wiedenhoft, H.; Winglee, K.; Humbert, O.; Edelstein, P.; Cosma, C.; Ramakrishnan, L. Drug Tolerance in Replicating Mycobacteria Mediated by a MacrophageInduced Efflux Mechanism. Cell, 2011, 145, 39-53.

[38] Weaver-Agostoni, J. Cluster headache. Am. Fam. Physician, 2013, 88, 122-128.

[39] Hoon, T.J.; Bauman, J.L.; Rodvold, K.A.; Gallestegui, J.; Hariman, R.J. The pharmacodynamic and pharmacokinetic differences of the $\mathrm{D}$ - and L-isomers of verapamil: Implications in the treatment of paroxysmal supraventricular tachycardia. Am. Heart J., 1986, 112, 396-403.

[40] Busse, D.; Templin, S.; Mikus, G.; Schwab, M.; Hofmann, U.; Eichelbaum, M.; Kivisto, K.T. Cardiovascular effects of (R)- and (S)-verapamil and racemic verapamil in humans: a placebo- controlled study. Eur. J. Clin. Pharmacol., 2006, 62, 613-619.

[41] Gupta, S.; Cohen, K.A.; Winglee, K.; Maiga, M.; Diarra, B.; Bishai, W.R. Efflux Inhibition with Verapamil Potentiates Bedaquiline in Mycobacterium tuberculosis. Antimicrob. Agents Chemother., 2014, 58, 574-576.

[42] Gupta, S.; Tyagi, S.; Bishai, W.R. Verapamil Increases the Bactericidal Activity of Bedaquiline against Mycobacterium tuberculosis in a Mouse Model. Antimicrob. Agents Chemother., 2015, 59, 673- 676.

[43] Andersen, C.L.; Holland, I.B.; Jacq, A. Verapamil, a $\mathrm{Ca}^{2+}$ channel inhibitor acts as a local anesthetic and induces the sigma $E$ dependent extra-cytoplasmic stress response in $E$. coli. Biochimica et Biophysica Acta (BBA) - Biomembranes, 2oo6, 1758, 1587-1595.

[44] Hait, W. N., Ed.; In Drug Resistance; Kluwer Academic Publishers: Boston, 1996.

[45] Jaszczyszyn, A.; Gasiorowski, K.; Swiatek, P.; Malinka, W.; Cieslik-Boczula, K.; Petrus, J.; Czarnik-Matusewicz, B. Chemical structure of phenothiazines and their biological activity. Pharmacol. Rep., 2012, 64, 16-23. 
[46] Weiss, B.; Prozialeck, W.C.; Wallace, T.L. Interaction of drugs with calmodulin. Biochemical, pharmacological and clinical implications. Biochem. Pharmacol., 1982, 31, 2217-2226.

[47] Daniel, W.A. Mechanisms of cellular distribution of psychotropic drugs. Significance for drug action and interactions. Prog. Neuro-psychopharmacol. Biol. Psychiat., 2003, 27, 65-73.

[48] Kornhuber, J.; Henkel, A.W.; Groemer, T.W.; Stadtler, S.; Welzel, O.; Tripal, P.; Rotter, A.; Bleich, S.; Trapp, S. Lipophilic cationic drugs increase the permeability of lysosomal membranes in a cell culture system. J. Cell. Physiol., 2010, 224, 152-164.

[49] Amaral, L.; Boeree, M.J.; Gillespie, S.H.; Udwadia, Z.F.; van Soolingen, D. Thioridazine cures extensively drug-resistant tuberculosis (XDR-TB) and the need for global trials is now! Int. J. Antimicrob. Agents, 2010, 35, 524-526.

[50] Abbate, E.; Vescovo, M.; Natiello, M.; Cufre, M.; Garcia, A.; Gonzalez Montaner, P.; Ambroggi, M.; Ritacco, V.; van Soolingen, D. Successful alternative treatment of extensively drug-resistant tuberculosis in Argentina with a combination of linezolid, moxifloxacin and thioridazine. J. Antimicrob. Chemother., 2012, 67, 473-477.

[51] Amaral, L.; Viveiros, M. Why thioridazine in combination with antibiotics cures extensively drug-resistant Mycobacterium tuberculosis infections. Int. J. Antimicrob. Agents, 2012, 39, 376-380.

[52] Bettencourt, M.V.; Bosne-David, S.; Amaral, L. Comparative in vitro activity of phenothiazines against multidrug-resistant Mycobacterium tuberculosis. Int. J. Antimicrob. Agents, 2000, 16, 69-71.

[53] Dutta, N.K.; Mehra, S.; Kaushal, D. A Mycobacterium tuberculosis sigma factor network responds to cell-envelope damage by the promising anti-mycobacterial thioridazine. PLoS One, 2010, 5, e10069.

[54] Amaral, L.; Molnar, J. Mechanisms by which thioridazine in combination with antibiotics cures extensively drug-resistant infections of pulmonary tuberculosis. In Vivo, 2014, 28, 267-271.

[55] de Keijzer, J.; Mulder, A.; de Haas, P.E.; de Ru, A.H.; Heerkens, E.M.; Amaral, L.; van Soolingen, D.; van Veelen, P.A. Thioridazine Alters the Cell-Envelope Permeability of Mycobacterium tuberculosis. J. Proteome Res., 2016, 15, 1776-1786.

[56] Crowle, A.J.; Douvas, G.S.; May, M.H. Chlorpromazine: a drug potentially useful for treating mycobacterial infections. Chemotherapy, 1992, 38, 410-419.

[57] Smith, D. A., Ed.; In Metabolism, Pharmacokinetics and Toxicity of Functional groups. Impact of the building blocks of medicinal chemistry on ADMET; Royal Society of Chemistry: Cambridge, 2010.

[58] Martins, M.; Viveiros, M.; Couto, I.; Amaral, L. Targeting human macrophages for enhanced killing of intracellular XDR-TB and MDR-TB. Int. J. Tuberc. Lung Dis., 2009, 13, 569-573.

[59] Amaral, L.; Martins, A.; Molnar, J.; Kristiansen, J.E.; Martins, M.; Viveiros, M.; Rodrigues, L.; Spengler, G.; Couto, I.; Ramos, J.; Dastidar, S.; Fanning, S.; McCusker, M.; Pages, J.M. Phenothiazines, bacterial efflux pumps and targeting the macrophage for enhanced killing of intracellular XDRTB. In Vivo, 2010, 24, 409-424. 
[6o] Dutta, N.K.; Karakousis, P.C. Thioridazine for treatment of tuberculosis: promises and pitfalls. Tuberculosis (Edinb), 2014, 94, 708-711.

[61] Rodrigues, L.; Ainsa, J.A.; Amaral, L.; Viveiros, M. Inhibition of drug efflux in mycobacteria with phenothiazines and other putative efflux inhibitors. Recent. Pat. Antiinfect. Drug Discov., 2011, 6, 118-127.

[62] Rodrigues, L.; Wagner, D.; Viveiros, M.; Sampaio, D.; Couto, I.; Vavra, M.; Kern, W.V.; Amaral, L. Thioridazine and chlorpromazine inhibition of ethidium bromide efflux in Mycobacterium avium and Mycobacterium smegmatis. J. Antimicrob. Chemother., 2008, 61, 1076-1082.

[63] Okumura, Y.; Narukawa, M.; Iwasaki, Y.; Ishikawa, A.; Matsuda, H.; Yoshikawa, M.; Watanabe, T. Activation of TRPV1 and TRPA1 by black pepper components. Biosci. Biotechnol. Biochem., 2010, 74, 1068-1072.

[64] Meghwal, M.; Goswami, T.K. Piper nigrum and piperine: an update. Phytother. Res., 2013, 27, 1121-1130.

[65] Ahmad, N.; Fazal, H.; Abbasi, B.H.; Farooq, S.; Ali, M.; Khan, M. A. Biological role of Piper nigrum L. (Black pepper): A review. Asian Pacific J. Trop. Biomed., 2012, 2, S1945-S1953.

[66] Bhardwaj, R. K.; Glaeser, H.; Becquemont, L.; Klotz, U.; Gupta, S. K.; Fromm, M.F. Piperine, a major constituent of black pepper, inhibits human P-glycoprotein and CYP3A4. J. Pharmacol. Exp. Ther., 2002, 302, 645-650.

[67] Singh, I.P.; Choudhary, A. Piperine and Derivatives: Trends in Structure-Activity Relationships. Curr. Top. Med. Chem., 2015, 15, 1722-1734.

[68] Kumar, A.; Khan, I.A.; Koul, S.; Koul, J.L.; Taneja, S.C.; Ali, I.; Ali, F.; Sharma, S.; Mirza, Z.M.; Kumar, M.; Sangwan, P.L.; Gupta, P.; Thota, N.; Qazi, G.N. Novel structural analogues of piperine as inhibitors of the NorA efflux pump of Staphylococcus aureus. $J$. Antimicrob. Chemother., 2008, 61, 1270-1276.

[69] Khan, I.A.; Mirza, Z.M.; Kumar, A.; Verma, V.; Qazi, G.N. Piperine, a Phytochemical Potentiator of Ciprofloxacin against Staphylococcus aureus. Antimicrob. Agents Chemother., 2005, 50, 810-812.

[70] Koul, S.; Koul, J.L.; Taneja, S.C.; Dhar, K.L.; Jamwal, D.S.; Singh, K.; Reen, R.K.; Singh, J. Structure-activity relationship of piperine and its synthetic analogues for their inhibitory potentials of rat hepatic microsomal constitutive and inducible cytochrome $\mathrm{P} 450$ activities. Bioorg. Med. Chem., 2000, 8, 251-268.

[71] Stermitz, F.R.; Lorenz, P.; Tawara, J.N.; Zenewicz, L.A.; Lewis, K. Synergy in a medicinal plant: antimicrobial action of berberine potentiated by 5 '-methoxyhydnocarpin, a multidrug pump inhibitor. Proc. Natl. Acad. Sci. USA, 2000, 97, 1433-1437.

[72] Tegos, G.; Stermitz, F.R.; Lomovskaya, O.; Lewis, K. Multidrug Pump Inhibitors Uncover Remarkable Activity of Plant Antimicrobials. Antimicrob. Agents Chemother., 2002, 46, 3133-3141.

[73] Wojtyczka, R.D.; Dziedzic, A.; Kepa, M.; Kubina, R.; Kabala-Dzik, A.; Mularz, T.; Idzik, D. Berberine enhances the antibacterial activity of selected antibiotics against coagulase-negative Staphylococcus strains in vitro. Molecules, 2014, 19, 6583-6596. 
[74] Zhou, X.; Ye, X.; He, L.; Zhang, S.; Wang, R.; Zhou, J.; He, Z. In vitro characterization and inhibition of the interaction between ciprofloxacin and berberine against multidrugresistant Klebsiella pneumoniae. J. Antibiot., 2016, 10, 741-746.

[75] Wang, Y.; Fu, H.; Li, Y.; Jiang, J.; Song, D. Synthesis and biological evaluation of 8substituted berberine derivatives as novel antimycobacterial agents. Acta Pharmaceut. Sinica $B$, 2012, 2, 581-587.

[76] Liu, C.; Zheng, Y.; Zhang, Y.; Long, X. Research progress on berberine with a special focus on its oral bioavailability. Fitoterapia, 2016, 109, 274-282.

[77] Fu, L.; Liang, Y.; Deng, L.; Ding, Y.; Chen, L.; Ye, Y.; Yang, X.; Pan, Q. Characterization of tetrandrine, a potent inhibitor of Pglycoprotein-mediated multidrug resistance. Cancer Chemother. Pharmacol., 2004, 53, 349-356.

[78] Henry, J. P.; Scherman, D. Radioligands of the vesicular monoamine transporter and their use as markers of monoamine storage vesicles. Biochem. Pharmacol., 1989, 38, 23952404.

[79] Login, I. S.; Judd, A. M.; Cronin, M. J.; Yasumoto, T.; MacLeod, R. M. Reserpine is a calcium channel antagonist in normal and GH3 rat pituitary cells. Am. J. Physiol., 1985, 248, E15-9.

[80] Louw, G.E.; Warren, R.M.; Gey, V.P.; Leon, R.; Jimenez, A.; Hernandez-Pando, R.; McEvoy, C. R. E.; Grobbelaar, M.; Murray, M.; van Helden, P.D.; Victor, T.C. Rifampicin reduces susceptibility to ofloxacin in rifampicin-resistant Mycobacterium tuberculosis through Efflux. Am. J. Respir. Crit. Care Med., 2011, 184, 269-276.

[81] Pule, C.M.; Sampson, S.L.; Warren, R.M.; Black, P.A.; van Helden, P.D.; Victor, T.C.; Louw, G.E. Efflux pump inhibitors: targeting mycobacterial efflux systems to enhance TB therapy. J. Antimicrob. Chemother., 2016, 71, 17-26.

[82] Pasca, M.R.; Guglierame, P.; De Rossi, E.; Zara, F.; Riccardi, G. mmpL7 Gene of Mycobacterium tuberculosis Is Responsible for Isoniazid Efflux in Mycobacterium smegmatis. Antimicrob. Agents Chemother., 2005, 49, 4775-4777.

[83] Viveiros, M.; Portugal, I.; Bettencourt, R.; Victor, T.C.; Jordaan, A. M.; Leandro, C.; Ordway, D.; Amaral, L. Isoniazid-induced transient high-level resistance in mycobacterium tuberculosis. Antimicrob. Agents Chemother., 2002, 46, 2804-2810.

[84] Zhang, Y.; Permar, S.; Sun, Z. Conditions that may affect the results of susceptibility testing of Mycobacterium tuberculosis to pyrazinamide. J. Med. Microbiol., 2oo2, 51, 42-49. [85] Klyachko, K.A.; Schuldiner, S.; Neyfakh, A.A. Mutations affecting substrate specificity of the Bacillus subtilis multidrug transporter Bmr. J. Bacteriol., 1997, 179, 2189-2193.

[86] Grossman, T.H.; Shoen, C.M.; Jones, S.M.; Jones, P.L.; Cynamon, M.H.; Locher, C.P. The efflux pump inhibitor timcodar improves the potency of antimycobacterial agents. Antimicrob. Agents Chemother., 2014, 59, 1534-1541.

[87] Mullin, S.; Mani, N.; Grossman, T.H. Inhibition of antibiotic efflux in bacteria by the novel multidrug resistance inhibitors biricodar (VX-710) and timcodar (VX-853). Antimicrob. Agents Chemother., 2004, 48, 4171-4176.

[88] Abdallah, H.M.; Al-Abd, A.; El-Dine, R.; El-Halawany, A. P-glycoprotein inhibitors of natural origin as potential tumor chemo-sensitizers: A review. J. Adv. Res., 2014, 6, 45-62.

[89] Lomovskaya, O.; Bostian, K.A. Practical applications and feasibility of efflux pump inhibitors in the clinic--a vision for applied use. Biochem. Pharmacol., 2006, 71, 910-918. 
[90] Balganesh, M.; Dinesh, N.; Sharma, S.; Kuruppath, S.; Nair, A.V.; Sharma, U. Efflux Pumps of Mycobacterium tuberculosis Play a Significant Role in Antituberculosis Activity of Potential Drug Candidates. Antimicrob. Agents Chemother., 2012, 56, 2643-2651.

[91] Lamers, R.P.; Cavallari, J.F.; Burrows, L.L. The efflux inhibitor phenylalaninearginine beta-naphthylamide (PAbetaN) permeabilizes the outer membrane of gramnegative bacteria. PLoS One, 2013, 8, e60666.

[92] Misra, R.; Morrison, K.D.; Cho, H.J.; Khuu, T. Importance of realtime assays to distinguish multidrug efflux pump-inhibiting and outer membrane-destabilizing activities in escherichia coli. $J$. Bacteriol., 2015, 197, 2479-2488.

[93] Bruhn, D.F.; Scherman, M.S.; Liu, J.; Scherbakov, D.; Meibohm, B.; Bottger, E.C.; Lenaerts, A.J.; Lee, R.E. In vitro and in vivo evaluation of synergism between antitubercular spectinamides and non-classical tuberculosis antibiotics. Sci. Rep., 2015, 5, 13985 .

[94] Thiebaut, F.; Tsuruo, T.; Hamada, H.; Gottesman, M.M.; Pastan, I.; Willingham, M.C. Cellular localization of the multidrug-resistance gene product P-glycoprotein in normal human tissues. Proc. Natl. Acad. Sci. USA, 1987, 84, 7735-7738.

[95] Subramanian, N.; Condic-Jurkic, K.; O’Mara, M.L. Structural and dynamic perspectives on the promiscuous transport activity of P-glycoprotein. Neurochem. Int., 2016, 98, 146-152.

[96] Montanari, F.; Ecker, G.F. Prediction of drug-ABC-transporter interaction--Recent advances and future challenges. Adv. Drug Deliv. Rev., 2015, 86, 17-26.

[97] Broccatelli, F.; Carosati, E.; Neri, A.; Frosini, M.; Goracci, L.; Oprea, T.I.; Cruciani, G. A novel approach for predicting P-glycoprotein (ABCB1) inhibition using molecular interaction fields. $J$. Med. Chem., 2011, 54, 1740-1751. 